\title{
Morphological and cytoskeletal alterations of nervous system tumor cells with different culturing methods
}

\author{
YOKO MATSUDA*, YOKO KAWAMOTO*, KIYOSHI TEDUKA, WEI-XIA PENG, \\ TETSUSHI YAMAMOTO, TOSHIYUKI ISHIWATA and ZENYA NAITO \\ Departments of Pathology and Integrative Oncological Pathology, Nippon Medical School, \\ 1-1-5 Sendagi, Bunkyo-ku, Tokyo 113-8602, Japan
}

Received November 16, 2010; Accepted January 17, 2011

DOI: 10.3892/ijo.2011.945

\begin{abstract}
Cell culture is one of the most important methods of research in molecular and cellular biology, and various culture systems have been developed, including two-dimensional (2D), three-dimensional (3D) and floating culture systems. In the present study, we examined morphological changes and different expression patterns of cytoskeletal proteins in three different types of nervous system tumor cells grown in 2D, 3D and floating cell cultures. A172, KG-1-C and IMR-32 cells showed marked morphological changes, depending on the cell culture methods. F-actin expression was clearly observed at the level of the cells nearest the plate surface in $2 \mathrm{D}$ and $3 \mathrm{D}$ cultures. On the other hand, expression of F-actin was weak in the floating culture system. $\alpha$-tubulin was detected in the cytoplasm of cells in 2D culture, but in floating and 3D cultures, $\alpha$-tubulin was expressed in the peripheral regions of spheres and spheroids. In conclusion, this study demonstrated that nervous system tumor cells showed different alterations in morphology, and different cytoskeletal protein expression patterns, depending on the culture methods.
\end{abstract}

\section{Introduction}

With the aim of mimicking in vivo situations or particular pathological conditions, various culture systems have been developed, including two-dimensional (2D), three-dimensional (3D), and floating culture systems $(1,2)$. Traditional tumor cell culture studies with 2D cultures have the advantages of ease and economy. Therefore, they have been commonly used to examine cancer cell biology and develop new drugs. However, tumor cells grown in 2D culture systems differ from those

Correspondence to: Dr Toshiyuki Ishiwata, Departments of Pathology and Integrative Oncological Pathology, Nippon Medical School, 1-1-5 Sendagi, Bunkyo-ku, Tokyo 113-8602, Japan

E-mail: ishiwata@nms.ac.jp

*Contributed equally

Key words: nervous system tumors, sphere, three-dimensional spheroidal culture, actin, tubulin grown in vivo; they have different morphology, cell-to-cell or cell-to-matrix adhesions, cellular differentiation patterns, and cellular behaviors $(3,4)$.

On the other hand, 3D cell culture systems were expected to mimic in vivo environments (5-7). Many types of 3D culture systems have been reported, including spheroid cultures, ex vivo cultures (8), multilayered postconfluent cell cultures (9), cells grown in an extracellular matrix gel (10), and co-cultures with fibroblasts or vascular endothelium (11). The recently developed NanoCulture plate, which has a microsquare pattern on the bottom of the plate, allows the formation of 3D spheroids without the need of any matrix or scaffold. Recently, we reported that several kinds of pancreatic cancer cell lines formed cell spheroids in NanoCulture plates (12). These cells showed alterations in the expression levels and localization of cytoskeletal proteins. The 3D spheroidal culture system with the NanoCulture plate is a useful method for cell imaging with phase contrast microscopy and confocal microscopy.

Furthermore, floating cultures in plates with a low-attachment surface permit the formation of spheres of several kinds of cells. It has been reported that neural stem cells and tumor stem cells grow in floating spheres in serum-free culture medium supplemented with epidermal growth factor (EGF) and/or basic fibroblast growth factor (bFGF) $(13,14)$. Therefore, the floating culture system is an important method for analyzing stem cells in vitro $(2,15)$.

Numerous genes and proteins have been found to be differently expressed in 2D, 3D, and floating culture models (16-19). Different culture methods can cause markedly different morphological changes in cells. Therefore, we hypothesized that these morphological changes might induce the alteration of cytoskeletal protein expression. In the present study, we examined morphological changes and different expression patterns of cytoskeletal proteins in three different types of nervous system tumors grown in 2D, floating, and 3D cell cultures.

\section{Materials and methods}

Materials. The following materials were purchased: NanoCulture 96-well plates (NCP-L-MS) and NanoCulture medium (NCM-M) from Scivax Corporation (Kanagawa, Japan); polyL-lysine coated 35-mm glass-bottom dishes from Matsunami 


\section{A}

$2 \mathrm{D}$ culture

Floating culture
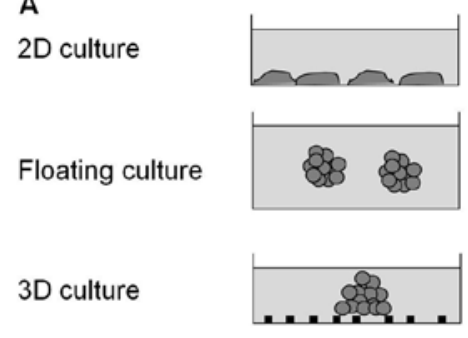

B
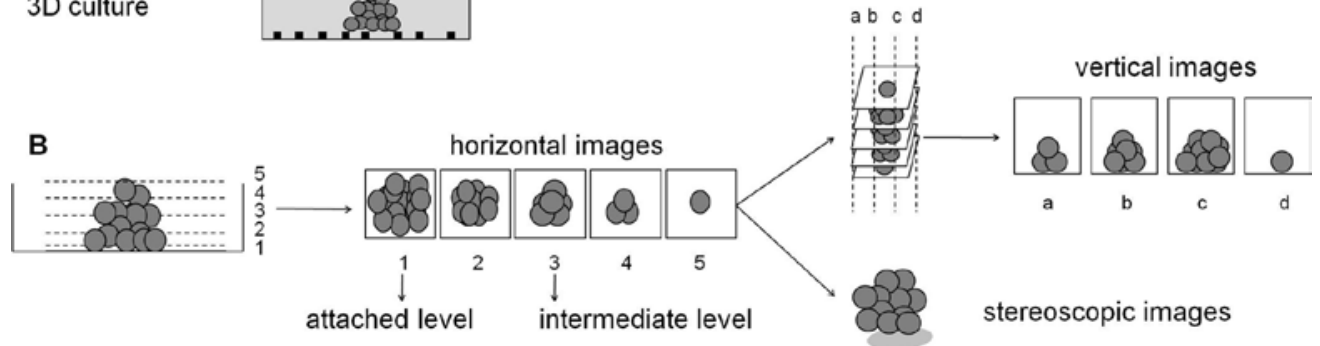

Figure 1. Schematics of cell culture and imaging methods. (A) Nervous system tumor cells were grown in (top) 2D cultures in glass-bottom dishes, (middle) floating cultures in plates with non-adherent surfaces, and (bottom) 3D cultures in NanoCulture plates. Stained cells were observed by confocal laser microscope. (B) (Left) Horizontal images were collected at $0.5 \mu \mathrm{m}$ intervals with a confocal laser microscope to create a stack on the Z axis (images 1-5). (Right) Images were then reconstructed to create vertical images (images a-d) and a stereoscopic image with image analysis software.

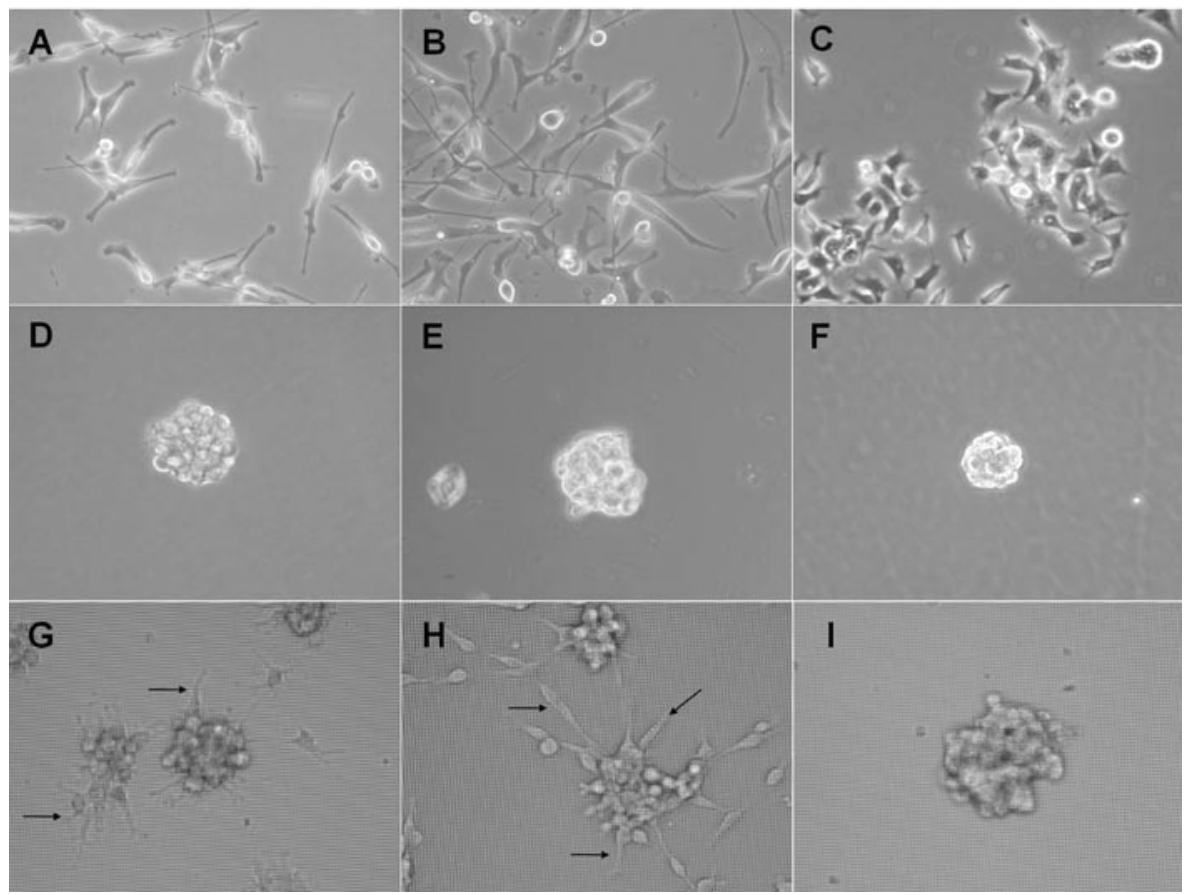

Figure 2. Phase contrast images of nervous system tumor cells. (A-C) In 2D cultures, all types of cells were attached to the dishes, and exhibited a sheet-like appearance, with spindle and polygonal cell shapes. (D-F) In floating cultures, the cells floated without attaching to the dish, and formed spheres. (G-I) In 3D cultures, the cells attached to the surface of the plate, and grew in a spheroid structure. (G, H, arrows) A172 and KG-1-C cells exhibited long cell projections around the spheres; (I) in contrast, IMR-32 cells showed no clear cell projections. A172 cells are shown in A, D and G; KG-1-C cells in B, E and H; IMR-32 cells in C, F, and I. Original magnification x 200 .

Glass Ind., Ltd. (Osaka, Japan); recombinant human EGF from Austral Biologicals (San Ramon, CA); recombinant human bFGF from ReproCell (Tokyo, Japan); 6-well ultra-low attachment surface plates from Corning Incorporated (Lowell, MA); rabbit polyclonal anti- $\alpha$-tubulin antibody (ab24246) from Abcam plc (Cambridge, UK); Alexa 568-labeled phalloidin and Alexa 488-labeled goat anti-rabbit IgG antibody from Invitrogen Corporation (Carlsbad, CA); and Vectashield H-1200 containing 4',6-diamidino-2-phenylindole-2HCl (DAPI) from Vector Lab., Inc. (Burlingame, CA). All other chemicals and reagents were purchased from Sigma-Aldrich Corporation (St. Louis, MO).

Nervous system tumor cell lines. Two glioma cell lines, A172 (human glioblastoma) and KG-1-C (human low grade glioma), and one human neuroblastoma cell line, IMR-32, were obtained from RIKEN BioResource Center (Ibaraki, Japan). The A172 cells were grown in RPMI-1640 medium with $10 \%$ fetal bovine 


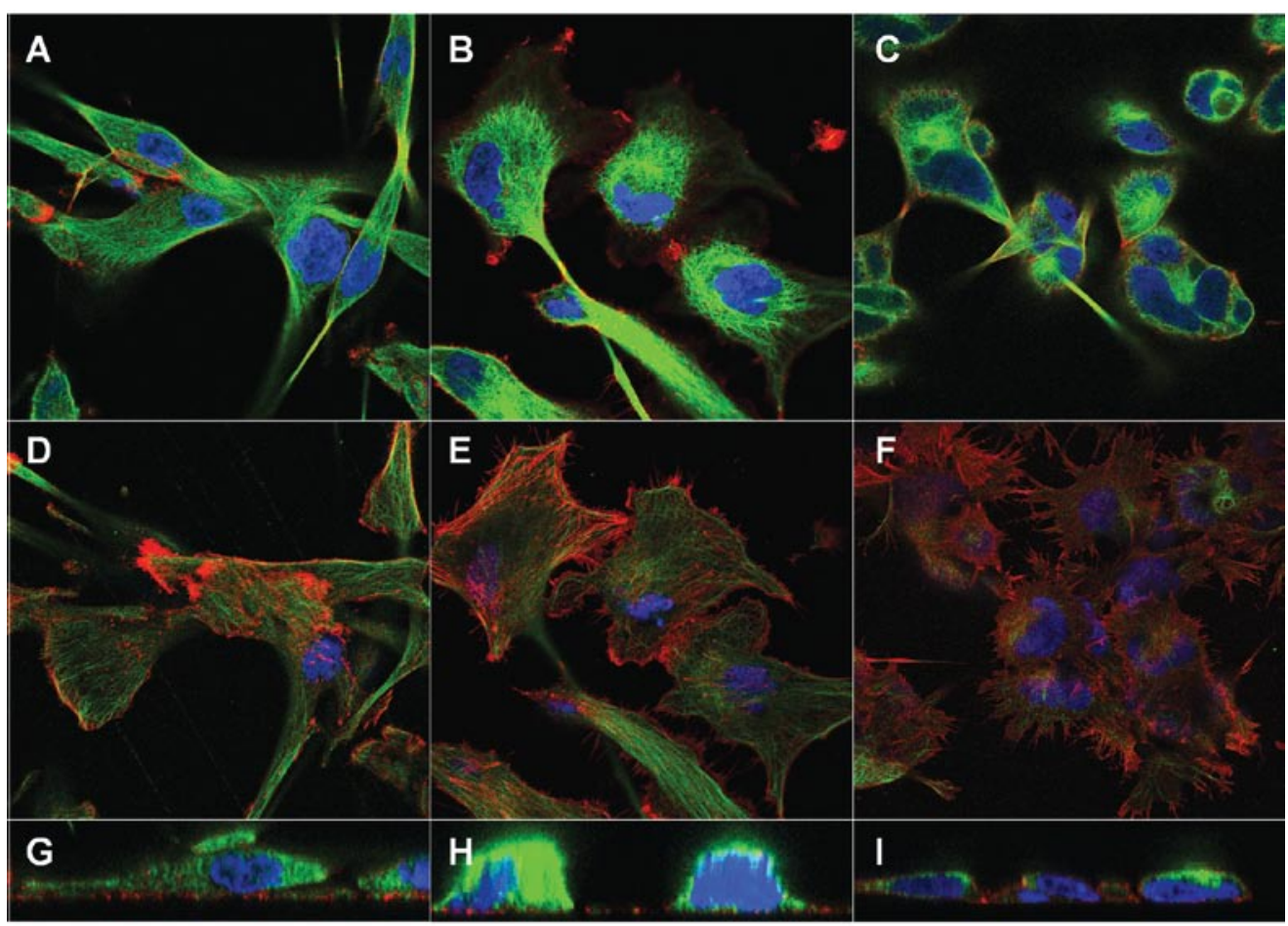

Figure 3. Expression of F-actin and $\alpha$-tubulin in 2D cultures. (A-C) In horizontal images, at the intermediate level of the $\mathrm{Z}$ axis, $\alpha$-tubulin (green) showed strong expression in the cytoplasm, while F-actin (red) was expressed only in the periphery of cells. (D-F) In horizontal images, at the level of the cells attached to the plates, F-actin expression was high, and stress fibers of F-actin were observed; $\alpha$-tubulin expression was weak. (G-I) In vertical images, F-actin expression was clearly observed in cells attached to the plate. A172 cells are shown in A, D and G; KG-1-C cells in B, E and H; IMR-32 cells in C, F and I; nuclei were stained blue. Original magnification x1000.

serum (FBS). The KG-1-C cells were cultured in DMEM medium with $20 \%$ FBS. The IMR-32 cells were cultured in MEM medium. All cells were cultured at $37^{\circ} \mathrm{C}$ under a humidified $5 \% \mathrm{CO}_{2}$ atmosphere.

Cell treatments. For 2D cell cultures, the nervous system tumor cells were suspended in growth medium with FBS and plated in $35-\mathrm{mm}$ glass-bottom dishes at a density of $1 \times 10^{5}$ cells $/ 3 \mathrm{ml}$ (Fig. 1A). For floating cultures, the cells were suspended in $3 \mathrm{ml}$ of growth medium with $20 \mathrm{ng} / \mathrm{ml}$ EGF and $20 \mathrm{ng} / \mathrm{ml}$ bFGF, and plated in ultra low-attachment surface plates $\left(2 \times 10^{3}\right.$ cells $\left./ 3 \mathrm{ml}\right)$. For 3D cell cultures, the cells were suspended in $0.1 \mathrm{ml}$ of NanoCulture medium with $10 \%$ FBS and plated in the NanoCulture plate $\left(1 \times 10^{4}\right.$ cells $\left./ 0.1 \mathrm{ml}\right)$. All cells were incubated for $72 \mathrm{~h}$ at $37^{\circ} \mathrm{C}$ in a humidified $5 \% \mathrm{CO}_{2}$ atmosphere.

Labeling for $\alpha$-tubulin and filamentous (F)-actin. The cells were fixed in a $4 \%$ paraformaldehyde solution for $15 \mathrm{~min}$, for 2D cultures, or $20 \mathrm{~min}$, for floating and 3D cultures, at room temperature. Fixed cells were incubated overnight at $4^{\circ} \mathrm{C}$ with a rabbit anti- $\alpha$-tubulin antibody (1:100 dilution). After incubation with Alexa 488-labeled anti-rabbit IgG antibody (1:1000 dilution) and Alexa 568-labeled phalloidin (1:50 dilution), the dishes were mounted in Vectashield $\mathrm{H}-1200$ that contained DAPI. The labeled $\alpha$-tubulin and F-actin proteins were visualized with a Digital Eclipse C1 TE2000-E microscope (Nikon Instech Co., Ltd., Tokyo, Japan). Fluorescence images were acquired with a Digital Eclipse C1 TE2000-E confocal microscope and analyzed with the control software EZ-C1 (Nikon Instech Co., Ltd.). The confocal settings, including the laser power and detector sensitivity, were constant for the acquisition of all images. Horizontal images were collected at $0.5 \mu \mathrm{m}$ intervals with a laser to create a stack on the $\mathrm{Z}$ axis; these were then used to reconstruct vertical stereoscopic images with the image analyzing software, Volocity (Improvision, Coventry, UK, Fig. 1B). A phase contrast image of the cells was examined with a Nikon Eclipse TE2000-U microscope.

\section{Results}

Cell morphologies. In 2D cultures, A172, KG-1-C, and IMR-32 cells were attached to dishes and exhibited a sheet-like appearance with spindle to polygonal cell shapes (Fig. 2A-C). In floating cultures, all cell types floated unattached to the dishes and formed spheres (Fig. 2D-F). In 3D cultures, cells were attached to the surfaces of plates, but grew in spheroid structures (Fig. 2G-I); the A172 and KG-1-C cells exhibited long cell projections around the spheres (Fig. $2 \mathrm{G}$ and $\mathrm{H}$, arrows), but IMR-32 showed no clear cell projections (Fig. 2I).

Cytoskeletal proteins are considered to be important regulators of cellular morphology and structure $(20,21)$. Therefore, we compared the expression patterns of cytoskeletal proteins in nervous system tumor cells under 2D, floating, and 3D culture conditions.

Expression patterns of $F$-actin and $\alpha$-tubulin in $2 D$ culture. In horizontal images of 2D cultures, at the intermediate level on the $\mathrm{Z}$ axis (Fig. 1B), $\alpha$-tubulin showed strong expression and formed fine fibers in the cytoplasm, but F-actin expression was weak and localized only to the periphery of cells (Fig. 3A-C). 


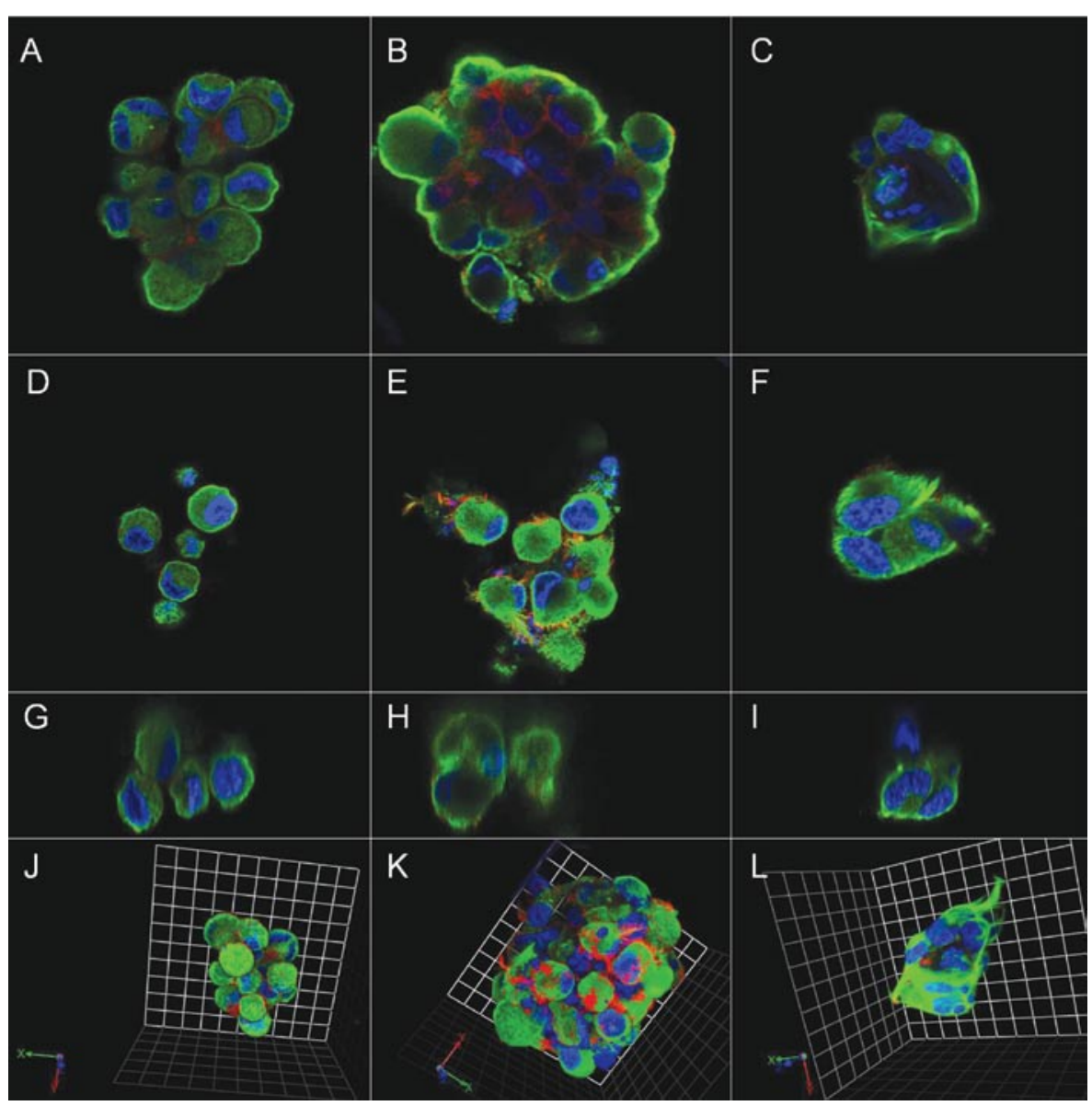

Figure 4. Expression of $\alpha$-tubulin and F-actin in floating cultures. (A-C) In horizontal images, at the intermediate level of the $\mathrm{Z}$ axis, $\alpha$-tubulin (green) was highly expressed, particularly in the periphery of spheres; F-actin (red) was expressed at the cell-to-cell junctions of spheres. (D-F) In horizontal images, at the lower level of the Z-axis of cells floating in the low-attachment plates, $\alpha$-tubulin expression was detected in all cells. (G-I) In vertical images reconstructed with computer software, sphere formation was not clearly observed, and $\alpha$-tubulin was strongly localized at the periphery of the cells. (J-L) In stereoscopic images reconstructed with computer software, sphere formation was clearly observed. A172 cells are shown in A, D, G and J; KG-1-C cells in B, E, H and K; IMR-32 cells in C, F, I and L; nuclei are stained blue. Original magnification x1000.

In horizontal images, at the level of cells attached to the dish (Fig. 1B), F-actin expression was high, and stress fibers of F-actin were clearly observed, but $\alpha$-tubulin expression was weak (Fig. 3D-F). In vertical images, at the level of cells attached to the surface of the dish, F-actin expression was clearly observed (Fig. 3G-I). Both horizontal and vertical images showed that F-actin was highly expressed at the surface attached to the dish, and $\alpha$-tubulin was abundantly expressed in the cytoplasm of cells at unattached sites.

Expression patterns of $F$-actin and $\alpha$-tubulin in floating cultures. In horizontal images of floating cultures, at the intermediate level of the $\mathrm{Z}$ axis, the maximum area of the sphere could be observed. In this region, $\alpha$-tubulin was highly expressed, particularly in the sphere periphery, and F-actin was expressed at the cell-to-cell junctions of spheres (Fig. 4A-C). In horizontal images, at the lower level of the $\mathrm{Z}$-axis of cells floating in the low-attachment plates, sphere formation was not clearly observed, but $\alpha$-tubulin expression was detected in all cells (Fig. 4D-F). F-actin expression was detected at the cell-to-cell junctions of spheres. In vertical images reconstructed with computer software, sphere formation and F-actin localization were not clearly observed due to the attenuation of the laser (Fig. 4G-I).
However, $\alpha$-tubulin was clearly localized to the periphery of cells. Stereoscopic images reconstructed with computer software allowed the clear detection of sphere formation (Fig. 4J-L). F-actin and $\alpha$-tubulin localization on the surface of the sphere could be observed. Expression of F-actin was observed at the cell-to-cell junctions on the surfaces of spheres.

Expression patterns of $F$-actin and $\alpha$-tubulin in $3 D$ spheroidal cultures. In horizontal images of 3D spheroids, at the intermediate level of the $\mathrm{Z}$ axis, $\alpha$-tubulin expression was high in the periphery, and F-actin expression was observed at the cell-to-cell junctions (Fig. 5A-C). In horizontal images at the level of cells attached to the plate, the grid structures of the NanoCulture plate could be observed, and F-actin expression was markedly increased (Fig. 5D-F). In A172 cells, F-actin was diffusely localized throughout the spheroids, and it was colocalized with $\alpha$-tubulin at the periphery of the spheroids (Fig. 5D). In KG-1-C cells, F-actin was colocalized with $\alpha$-tubulin at the periphery of the cells (Fig. 5E). Vertical images showed that F-actin expression was observed at the level of cells attached to the surface of the plate, but the morphological changes observed in the upper level cells were not clearly observed here, due to the attenuation of the laser (Fig. 5G-I). Stereoscopic 


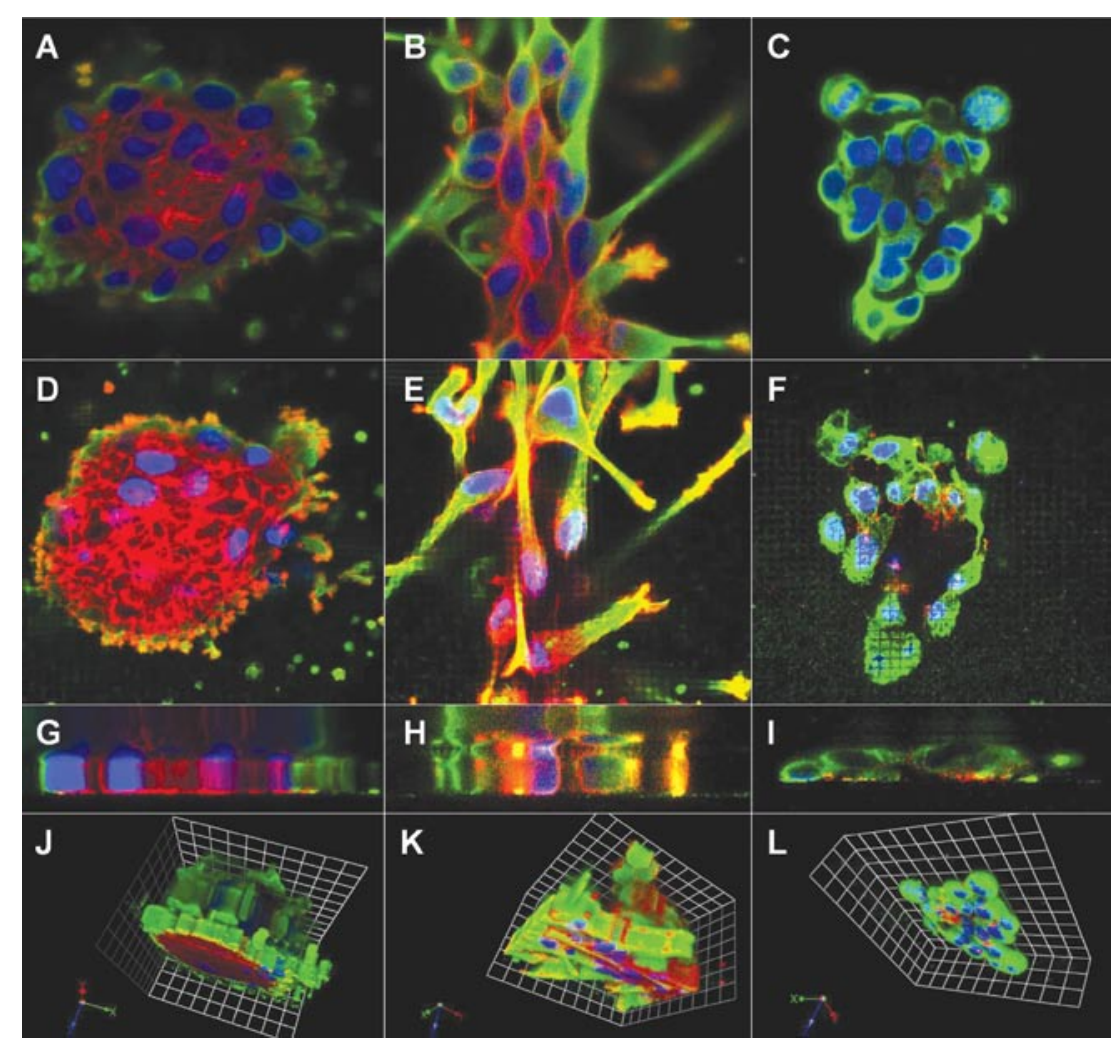

Figure 5. Expression of $\alpha$-tubulin and F-actin in 3D cultures. (A-C) In horizontal images, at the intermediate level of the $\mathrm{Z}$ axis, $\alpha$-tubulin (green) was highly expressed in the periphery of spheroids and F-actin (red) was expressed at the cell-to-cell junctions of spheroids. (D-F) In horizontal images, at the level of cells attached to the plate, grid structures of the NanoCulture plate were observed and F-actin expression markedly increased. (G-I) In vertical images, F-actin expression was observed in cells attached to the plate. (J-L) Stereoscopic images reconstructed with computer software showed F-actin expression in the center of the spheroids. A172 cells are shown in A, D, G and J; KG-1-C cells in B, E, H and K; and IMR-32 cells in C, F, I and L; nuclei are stained blue. Original magnification $x 1000$.

images showed that F-actin expression was high in the center of spheroids and in the cells attached to the surface of the plate (Fig. 5J-L). F-actin expression was markedly elevated in 3D cultures compared with 2D and floating cultures.

\section{Discussion}

In the present study, A172, KG-1-C, and IMR-32 cells showed marked morphological changes, depending on the cell culture methods. To examine underlying mechanisms of the changes in cell morphology, we analyzed the expression patterns of cytoskeletal proteins, including F-actin and $\alpha$-tubulin. F-actin expression was clearly observed at the level of the cells nearest the plate surface in 2D and 3D cultures. On the other hand, expression of F-actin was weak in the floating culture system. F-actin is anchored to the cell membrane, and the sites where this anchoring occurs are frequently connected to structures outside the cells. Therefore, differences in cell-to-cell interactions and cell-to-extracellular matrix interactions among the culture systems might alter the expression levels and patterns of actin fibers.

The expression pattern of $\alpha$-tubulin in nervous system tumor cells was also different in different culture systems. In 2D culture, $\alpha$-tubulin was detected in the cytoplasm of cells, but in floating and 3D cultures, $\alpha$-tubulin was expressed in the peripheral regions of spheres and spheroids. Tubulin forms microtubules and centrosomes; it is also essential for many vital cellular processes, including intracellular transport, metabolism, and cell division. The differences in $\alpha$-tubulin expression observed among culture systems might be important for studying tumor cell behaviors.

Previous studies have shown that many genes and proteins were expressed differently in the same cell lines cultured in 2D, 3D, and floating systems (16-19). Furthermore, floating cultures in plates with non-adherent surfaces have been widely used to investigate the roles of stem cells (15).

In the present study, vertical and stereoscopic image analyses were found to be equally useful for morphological analyses. Horizontal images allowed the analysis of protein expression at several levels. Vertical and stereoscopic images provided many more levels of analysis, for example morphological changes and expression patterns of proteins in spheres and spheroids. In vertical images, the upper levels of cell structures were incomprehensible due to the attenuation of the laser. However, we could examine the localization of F-actin and $\alpha$-tubulin at the bottom surfaces of cell structures. Stereoscopic images, constructed with image analysis software, provided better images and facilitated the analysis of compositional differences between cell structures. In conclusion, this study demonstrated that nervous system tumor cells showed different alterations in morphology, and different cytoskeletal protein expression patterns, depending on the culture methods. The results also 
indicated that stereoscopic analysis with image analysis software could provide a large amount of information on spheres and spheroids.

\section{Acknowledgements}

This study was supported by grants (no. S0801035, to Z. Naito) from the Ministry of Education, Culture, Sports, Science, and Technology (MEXT), Japan.

\section{References}

1. Lund AW, Yener B, Stegemann JP and Plopper GE: The natural and engineered $3 \mathrm{D}$ microenvironment as a regulatory cue during stem cell fate determination. Tissue Eng Part B Rev 15: 371-380, 2009.

2. Sasaki R, Aoki S, Yamato M, Uchiyama H, Wada K, Ogiuchi H, Okano T and Ando T: A protocol for immunofluorescence staining of floating neurospheres. Neurosci Lett 479: 126-127, 2010.

3. Nelson CM and Bissell MJ: Of extracellular matrix, scaffolds, and signaling: tissue architecture regulates development, homeostasis, and cancer. Annu Rev Cell Dev Biol 22: 287-309, 2006.

4. Petersen MC, Lazar J, Jacob HJ and Wakatsuki T: Tissue engineering: a new frontier in physiological genomics. Physiol Genomics 32: 28-32, 2007.

5. Yamada KM and Cukierman E: Modeling tissue morphogenesis and cancer in 3D. Cell 130: 601-610, 2007.

6. Vincan E, Brabletz T, Faux MC and Ramsay RG: A human three-dimensional cell line model allows the study of dynamic and reversible epithelial-mesenchymal and mesenchymal-epithelial transition that underpins colorectal carcinogenesis. Cells Tissues Organs 185: 20-28, 2007.

7. Schagemann JC, Mrosek EH, Landers R, Kurz H and Erggelet C: Morphology and function of ovine articular cartilage chondrocytes in 3-d hydrogel culture. Cells Tissues Organs 182: 89-97, 2006.

8. Sakai T, Larsen M and Yamada KM: Fibronectin requirement in branching morphogenesis. Nature 423: 876-881, 2003.

9. Padron JM, van der Wilt CL, Smid K, Smitskamp-Wilms E, Backus HH, Pizao PE, Giaccone G and Peters GJ: The multilayered postconfluent cell culture as a model for drug screening. Crit Rev Oncol Hematol 36: 141-157, 2000.
10. Yamada M, Moritoh C, Kawaguchi M and Okigaki T: Growth, morphology, function, and morphogenetic properties of rat renal glomerular epithelial cells in vitro: effects of retinyl acetate. Eur J Cell Biol 49: 252-258, 1989.

11. Smalley KS, Lioni M and Herlyn M: Life isn't flat: taking cancer biology to the next dimension. In Vitro Cell Dev Biol Anim 42: 242-247, 2006

12. Matsuda Y, Ishiwata T, Kawamoto Y, Kawahara K, Peng W, Yamamoto T and Naito Z: Morphological and cytoskeletal changes of pancreatic cancer cells in three-dimensional spheroidal culture. Mol Med Morphol (In Press).

13. Singh SK, Clarke ID, Terasaki M, Bonn VE, Hawkins C, Squire J and Dirks PB: Identification of a cancer stem cell in human brain tumors. Cancer Res 63: 5821-5828, 2003.

14. Singh SK, Hawkins C, Clarke ID, Squire JA, Bayani J, Hide T, Henkelman RM, Cusimano MD and Dirks PB: Identification of human brain tumour initiating cells. Nature 432: 396-401, 2004.

15. Reynolds BA and Weiss S: Generation of neurons and astrocytes from isolated cells of the adult mammalian central nervous system. Science 255: 1707-1710, 1992.

16. Qiang L, Yang Y, Ma YJ, Chen FH, Zhang LB, Liu W, Qi Q, $\mathrm{Lu} \mathrm{N}$, Tao L, Wang XT, You QD and Guo QL: Isolation and characterization of cancer stem like cells in human glioblastoma cell lines. Cancer Lett 279: 13-21, 2009.

17. Ghosh S, Spagnoli GC, Martin I, Ploegert S, Demougin P, Heberer $\mathrm{M}$ and Reschner A: Three-dimensional culture of melanoma cells profoundly affects gene expression profile: a high density oligonucleotide array study. J Cell Physiol 204: 522-531, 2005

18. Shimada M, Yamashita Y, Tanaka S, Shirabe K, Nakazawa K, Ijima H, Sakiyama R, Fukuda J, Funatsu K and Sugimachi K: Characteristic gene expression induced by polyurethane foam/ spheroid culture of hepatoma cell line, Hep G2 as a promising cell source for bioartificial liver. Hepatogastroenterology 54: 814-820, 2007

19. Takagi A, Watanabe M, Ishii Y, Morita J, Hirokawa Y, Matsuzaki T and Shiraishi T: Three-dimensional cellular spheroid formation provides human prostate tumor cells with tissue-like features. Anticancer Res 27: 45-53, 2007.

20. Kueh HY and Mitchison TJ: Structural plasticity in actin and tubulin polymer dynamics. Science 325: 960-963, 2009.

21. Albiges-Rizo C, Destaing O, Fourcade B, Planus E and Block MR: Actin machinery and mechanosensitivity in invadopodia, podosomes and focal adhesions. J Cell Sci 122: 3037-3049, 2009. 